\title{
EL DESARROLLO DEL APRENDIZAJE REFLEXIVO DESDE LA ASIGNATURA GENÉTICA MÉDICA
}

\author{
The development of reflective learning from the medical genetic subject
}

\author{
Midiel Marcos Mendoza, Lcdo. \\ Universidad de Sancti Spíritus José Martí \\ Pérez, Cuba \\ https://orcid.org/0000-0002-3503-6201 \\ mmendoza@uniss.edu.cu
}

\author{
Zuyen Fernández Caballero, Dr. C. \\ Universidad de Sancti Spíritus José Martí \\ Pérez, Cuba \\ https://orcid.org/0000-0001-5281-3650 \\ zuyen@uniss.edu.cu
}

\author{
Zaida González Fernández, Dr. C. \\ Universidad de Sancti Spíritus José Martí \\ Pérez, Cuba \\ https://orcid.org/0000-0003-2375-1280 \\ zgonzalez@uniss.edu.cu
}

Palabras claves: Aprendizaje, Reflexivo, Estrategia.

Recibido: 29 de octubre de 2018

Keywords: Learning, Reflexive, Strategy.

Aceptado: 20 de marzo de 2019

\section{RESUMEN}

El aprendizaje reflexivo garantiza la apropiación activa y creativa de los conocimientos preparando al estudiante para resolver los problemas de la profesión. El artículo presenta una estrategia didáctica dirigida al desarrollo del aprendizaje reflexivo en los estudiantes de segundo año de la carrera de Medicina de la Facultad de Ciencias Médicas de la provincia de Sancti Spíritus. Se realizó un estudio experimental en el período comprendido de enero de 2016 a julio de 2016. La población fue de 296 estudiantes que cursan la asignatura Genética Médica, y la muestra fue de un grupo (29). Se emplearon métodos teóricos: inductivo-deductivo, histórico-lógico y analítico-sintético; y empíricos: análisis documental, encuesta, y métodos estadísticos-matemáticos. Se constató que la concepción y formulación de los objetivos no facilita la orientación para alcanzarlos con éxito; además, de la tendencia a favorecer la asimilación reproductiva. La estrategia didáctica pretende el desarrollo del aprendizaje reflexivo. Ella permite, estimular el aprendizaje reflexivo, hace posible que el contenido, se estudie en sus contradicciones, facilita la búsqueda, el cuestionamiento y la puesta en práctica de procedimientos, Fomenta el vínculo teoría-práctica y concede una función protagónica a los estudiantes en su proceso de aprendizaje.

\section{ABSTRACT}

Reflexive learning guarantees the active and creative appropriation of knowledge by preparing the student to solve the problems of the profession. The paper aims to: design a didactic strategy aimed at the development of reflexive learning in the second year students of the medical school of the Faculty of Medical Sciences of the province of Sancti Spíritus An experimental study was carried out in the period of January From 2016 to July 2016. The population was 296 students attending Medical Genetics, and the sample was from a group (29). Theoretical methods were used: inductive-deductive, historical-logical and analytical-synthetic; And empirical: documentary analysis, survey, and statistical-mathematical methods. It was found that the design and formulation of the objectives did not facilitate the orientation to achieve them successfully; In addition, the tendency to favor the reproductive assimilation. The didactic strategy aims at the development of reflective learning. It allows, stimulates reflexive learning, makes it possible for content to be studied in its contradictions, facilitates the search, questioning and implementation of procedures. It fosters the theory-practice link and gives a leading role to students in their learning process. 


\section{INTRODUCCIÓN}

En el mundo actual, caracterizado por la globalización neoliberal, la revolución de los sistemas de información y comunicación, los rápidos y asombrosos avances tecnológicos, el deterioro del medio ambiente, entre otros, hace que las capacidades y la inteligencia humana se conviertan en recursos inagotables para el desarrollo y equilibrio de los pueblos; por lo que, constituye una obligación de la sociedad y de la escuela en particular invertir fuerzas en su desarrollo, y una vía fundamental es el aprendizaje.

De todas las instituciones en las que encarga la función educativa de la sociedad, le corresponde un papel estratégico a la escuela, en sus diferentes niveles de enseñanza desde la educación preescolar hasta la educación superior, ya que es en ella donde se dirige el proceso de enseñanza-aprendizaje. Una educación superior pertinente es aquella que establece de forma prioritaria una relación obligada y con carácter de ley entre su misión y las demandas sociales que se corresponden con ésta en cada momento histórico, social y cultural concreto.

El estudio del aprendizaje se ha convertido en el centro de atención de psicólogos, pedagogos y sociólogos los que subrayan, desde concepciones y enfoques psicopedagógicos relativamente dispares, el problema del aprendizaje humano, y el papel de la actividad en su desarrollo. En el estudio son considerados antecedentes a los investigadores que han dirigido su atención al análisis del aprendizaje destacándose Viera T, T (2003), Torrance, E.P. (1999), Martínez LL, M (1998), Castellanos, D. (2003), Addine F, F. (2006), entre otros.

Los estudiantes de Medicina hoy son los médicos del mañana, por lo que no existe mejor inversión que la potenciación de sus posibilidades de desarrollo, y es a la escuela, y, por tanto, al personal docente a quienes les corresponde por historia y formación, guiar este proceso. El diseño curricular del sistema, por el afán de despachar información muchas veces, no deja espacio para la reflexión y el análisis de la información recibida. El proceso de enseñanza-aprendizaje debe ser menos informativo y más formativo.

Para el profesor lo más importante debe ser que el estudiante logre encontrar el sentido, su propio sentido, a lo que acontece y a la información que recibe, que piense y reflexione en el proceso de aprendizaje, para lo cual es imprescindible un proceso de aprendizaje que conlleve, en sí mismo, al gozo, la comprensión intuitiva y, sobre todo, al amor por el saber, por lo bello y hermoso de conocer.

En la realidad universitaria se pueden descubrir estudiantes con una actitud pasiva ante el estudio, que, en el mejor de los casos sólo escuchan al profesor y no participan como agentes activos en el proceso de su propio aprendizaje.

Ante esta situación se hace necesario se traslada una información y se obtiene una respuesta reproductiva; subordinándose el desarrollo intelectual de los estudiantes a la función instructiva, la cual, muchas veces, se forma con pobres pretensiones, constituyendo una barrera, para desarrollar la capacidad de pensar, razonar y actuar frente a nuevas situaciones, limitando la puesta en práctica de los conocimientos adquiridos y las habilidades profesionales requeridas para ello.

La problemática antes descrita se pone de manifiesto en la impartición de la asignatura Genética Médica en algunos estudiantes de segundo año de la carrera de Medicina.

La experiencia de los autores como docentes de la educación superior y especialmente de esta asignatura, le ha permitido constatar algunas insuficiencias relacionadas con la conducción del proceso docente que limitan la asunción de aprendizajes reflexivos en estos estudiantes. En esencia las limitaciones giran en torno a que no siempre se utilizan métodos productivos en los niveles de enseñanza precedentes lo que estimula el aprendizaje memorístico.

Los niveles de conocimientos, considerados base para el aprendizaje de algunas disciplinas de la especialidad, resultan insuficientes, este elemento compromete los niveles de precedencia necesarios para la adquisición del nuevo conocimiento, además, el desarrollo de las habilidades intelectuales que se necesita para el empleo de métodos productivos, no está al nivel que se necesita, por lo que cualquier intento de utilizarlos resulta limitado en el resultado.

Sobre la base de la idea de cómo contribuir a la solución del problema detectado, los autores se plantearon como objetivo: diseñar una estrategia didáctica dirigida al desarrollo del aprendizaje reflexivo en los estudiantes de segundo año de la carrera de Medicina de la Facultad de Ciencias Médicas de la provincia de Sancti Spíritus. 


\section{DESARROLLO}

En esta ponencia se exponen los resultados de una investigación en curso donde se sistematiza, sobre la base del el conocimiento precedente y el diagnóstico, la realidad educativa, para el diseño de una estrategia didáctica dirigida al desarrollo del aprendizaje reflexivo.

La metodología utilizada incluyó el desarrollo de una primera etapa de un estudio experimental en el período comprendido entre enero de 2016 a julio de 2016 en la Facultad de Ciencias Médicas de Sancti Spíritus. La población estuvo conformada por la totalidad de estudiantes (296) que cursan la asignatura Genética Médica (2do año), en la etapa señalada y la muestra fue de un grupo (29), seleccionados por muestreo intencional no probabilístico

Los métodos teóricos utilizados fueron: analítico-sintético, inductivo-deductivo, análisis histórico-lógico, los que permitieron determinar:

Diferentes tendencias en el proceso de enseñanza-aprendizaje, que han prevalecido en la formación de médicos, desde la puesta en práctica del plan de estudio "A", hasta el vigente.

Los aportes que, acerca del aprendizaje, han sido ofrecidos por diferentes autores. Las dificultades fundamentales que se han presentado en la dirección del aprendizaje de los estudiantes en la asignatura Genética Médica.

El enfoque de sistema, para la determinación de los elementos y las relaciones esenciales dela estrategia didácticadirigida al desarrollo del aprendizaje reflexivo.

Del nivel empírico se utilizaron:

La observación pedagógica para determinar las principales insuficiencias e irregularidades que presentan los estudiantes en el aprendizaje reflexivo de la asignatura Genética Médica.

La encuesta, mediante cuestionarios, para determinar opiniones y puntos de vista de los estudiantes y los profesores acerca del proceso de enseñanza-aprendizaje de la Genética Médica en la formación de los médicos y la estimulación de su aprendizaje.

Análisis documental para la consulta a planes de estudio, Programas y orientaciones metodológicas.

La experimentación a partir de la variante de Pre-experimento, como modelo de diseño experimental, para valorar los resultados obtenidos en el aprendizaje de los estudiantes al aplicar la estrategia didáctica propuesta.

Métodos del nivel estadístico-matemático: permitieron valorar los resultados obtenidos en las diferentes etapas de la investigación. Las técnicas, como la comparación de proporciones y la prueba de rangos señalados y pares asociados de Wilcoxon, contribuirán a valorar el comportamiento de las variables experimentales. Dentro de estos métodos, se utilizaron los de la Estadística Descriptiva, para la confección de tablas de distribución de frecuencia y de gráficos.

\section{Resultados}

Luego de la consulta bibliográfica y la aplicación de los instrumentos que permitieron corroborar el estado actual en la realidad educativa, en los elementos referidos al aprendizaje reflexivo desde la asignatura Genética Médica, emerge la estrategia didáctica que orienta cómo transformar la realidad hasta alcanzar el estado ideal. La estrategia educativa fomenta el vínculo teoría-práctica, al considerar el trabajo con problemas profesionales relacionados con la actividad laboral de los estudiantes, en una concepción de aprendizaje apoyada en la actividad reflexiva de los estudiantes, la problematización y la motivación. Es contentiva de objetivos generales, etapas con sus objetivos específicos y acciones para cada una de las etapas.

\section{Discusión}

Para este análisis se tiene en cuenta el diagnóstico efectuado en la etapa de constatación de la investigación, el que se dirige a comprobar el estado del aprendizaje reflexivo de los estudiantes investigados de la carrera de Ciencias Médicas escogida, a partir de medir el comportamiento de los indicadores determinados para este tipo de aprendizaje. Abarca lo siguiente:

- Análisis de los programas de disciplinas y asignaturas para comprobar si su concepción favorece la estimulación del aprendizaje reflexivo.

- Exploración de la situación del aprendizaje reflexivo en los estudiantes de las carreras de Ciencias Médicas. 
- Observaciones a clases para comprobar si contribuyen a estimular el aprendizaje reflexivo.

Fueron analizados el programa de la Disciplina y la asignatura Genética Médica. Este análisis permite determinar que los programas, en sentido general, responden a las exigencias del plan de estudio " $D$ " y, por tanto, potencian el trabajo de los estudiantes en los componentes académico, laboral e investigativo, en estrecho vínculo.

No obstante, presentan dificultades, que deben ser tenidas en cuenta si se trata de estimular el aprendizaje reflexivo:

$\checkmark$ En ocasiones, la concepción y formulación de los objetivos no facilita que los estudiantes, puedan orientarse para alcanzarlos;

$\checkmark$ Continúa presente la tendencia a la asimilación reproductiva de los contenidos;

$\checkmark \quad$ No en todos los programas se logra que los objetivos tengan carácter formativo y que, en su formulación, expresen el resultado, la intención, el conocimiento y la vía para alcanzarlos;

$\checkmark \quad$ En las orientaciones metodológicas del programa, tanto de la disciplina como de la asignatura, no queda claro el enfoque metodológico para el logro de un aprendizaje reflexivo;

$\checkmark \quad$ Las precisiones de cada tema se limitan a informar superficialmente sobre algunos contenidos y orientar vagamente algunos aspectos a los que debe referirse el profesor al abordarlos;

$\checkmark$ No se caracterizan, con profundidad, los contenidos y no quedan suficientemente esclarecidas las contradicciones que, como parte del contenido, pueden ser abordadas para que, en estrecho vínculo con su actividad práctica, solucionen problemas profesionales;

$\checkmark \quad$ Limitaciones en la actividad reflexiva de los estudiantes, su motivación y el protagonismo en su aprendizaje;

$\checkmark \quad$ No existe un balance adecuado respecto a la propuesta de métodos reproductivos y productivos, prevalecen los primeros, lo que atenta contra la implicación productiva de los estudiantes en su aprendizaje y, por consiguiente, de lograr que su aprendizaje sea reflexivo;

$\checkmark \quad$ Al analizar el sistema de evaluación propuesto, se corroboró su carácter reproductivo;

$\checkmark \quad$ No son estimuladas formas de evaluación que permitan el intercambio y el análisis crítico de puntos de vista y opiniones;

$\checkmark$ En todos los programas revisados aparece indicada una amplia bibliografía que, si es orientada correctamente por el profesor y manejada adecuadamente por los estudiantes, puede contribuir a lograr preparación y dominio de la asignatura.

La aplicación de una encuesta a los 29 estudiantes del grupo 6 de segundo año, permite determinar las siguientes regularidades:

$\checkmark \quad$ Falta interés por el estudio y por poner en práctica formas de aprender que dependen, en gran medida, de su implicación;

$\checkmark \quad$ Los conocimientos precedentes son escasos y existen pocas posibilidades de utilización para desarrollar otros nuevos;

$\checkmark$ Emplean constantemente la memoria reproductiva;

$\checkmark$ Dificultades para la toma de notas y para la elaboración propia de los contenidos que se desarrollan;

$\checkmark \quad$ No siempre realizan el trabajo independiente con la profundidad que requieren las diferentes asignaturas.

La conducción adecuada del proceso de enseñanza aprendizaje supone comprenderlo como un proceso pedagógico que posea las características esenciales de éste, pero se distingue por ser mucho más sistemático, planificado, dirigido y específico por cuanto la interrelación maestro - alumno, deviene en un accionar didáctico mucho más directo, cuyo único fin es el desarrollo integral de la personalidad de los educandos. En la revisión bibliográfica realizada varios investigadores abordan esta temática relacionada con el aprendizaje reflexivo. Gilberto G (2002), propone diez principios a tener en cuenta para la creación de situaciones de enseñanza y aprendizaje desarrolladores entre los que se encuentran:

- La posibilidad de aprender a través de actividades desafiantes que despierten las motivaciones intrínsecas;

- La participación y solución en problemas reales, contextualizados, que permitan explorar, descubrir y hacer por cambiar la realidad;

- La transformación del estudiante de receptor en investigador y productor de información;

- La promoción del autoconocimiento, de la autovaloración y de la reflexión acerca del proceso de aprendizaje y la valorización de la autodirectividad y autoeducación como meta.

En Cuba, fue a partir de la década de 1990 que cobró mayor auge su estudio como respuesta a la necesidad de formar hombres reflexivos, críticos, creativos, con dominio de sí, como demanda la sociedad cubana que se construye. Recientemente, el seminario nacional para profesores, efectuado en el mes de noviembre de 2004, asumió el desarrollo 
de este aprendizaje como necesidad para lograr la transformación de los sujetos que aprenden. Promover la confrontación y ayudar a revelar contradicciones, a partir del contenido, son propuestas realizadas por Castellanos S, D y colaboradores (2003) para lograr el aprendizaje crítico-reflexivo.

Adquiere importancia la asunción del aprendizaje reflexivo al comprender la visión de algunos autores locales que muestran la necesidad de un proceso de aprendizaje que desencadena el desarrollo, el cual encierra una transformación individual que posibilita evolucionar el contexto educacional en el que se actúa, como resultado del enriquecimiento y actualización de los contenidos, métodos de la ciencia y valores, que se logra en la interacción de lo grupal e individual. Sobre la base de los resultados del diagnóstico y la consulta teórica realizada se decide diseñar una estrategia didáctica. La estrategia que se presenta es didáctica, al tener en cuenta la actividad del profesor para enseñar en unidad indisoluble con la actividad de los estudiantes para aprender.

Además, se ha considerado la complejidad del proceso que se analiza y lo factible que resulta la propuesta que, aplicada en el proceso de enseñanza-aprendizaje de la carrera de Ciencias Médicas, tenga en cuenta las características, las exigencias, las dimensiones y los indicadores del aprendizaje reflexivo determinados por los autores de este trabajo.

El dominio, por parte de los profesores, de cómo lograr un proceso de enseñanza-aprendizaje que satisfaga la necesidad de estimular el aprendizaje reflexivo permitirá darle solución a las insuficiencias que se presentan en la adquisición de conocimientos de la asignatura Genética Médica, como componente esencial para la integración de saberes en la solución de los problemas profesionales descritos en el modelo del profesional.

Además, que se prepara a los estudiantes, en función de un aprendizaje que permite su crecimiento personal y realización profesional, lo que favorece el enriquecimiento del proceso de enseñanza-aprendizaje que se desarrolla en las facultades de Ciencias Médicas, lo que indiscutiblemente garantiza la calidad del proceso.

Se seleccionó la asignatura Genética Médica, para la implementación de la estrategia; no obstante, cualquiera de las asignaturas del currículo puede ser objeto válido para este análisis, pues el diagnóstico aplicado, en la etapa de constatación, corroboró que las dificultades no eran exclusivas de una asignatura particular, sino que eran comunes a la formación de este profesional.

Los profesores deben estar conscientes de la necesidad de modificar el proceso de enseñanza-aprendizaje en función de estimular el aprendizaje reflexivo en los estudiantes. Para ello, deben incorporar, a su actividad docente con carácter sistemático y sistémico, los fundamentos teóricos y metodológicos, objeto de análisis. Concederles a los estudiantes la posición protagónica y productiva que les corresponde en el proceso de enseñanza-aprendizaje.

La estrategia didáctica diseñada persigue como objetivo: Estimular el aprendizaje reflexivo desde la asignatura Genética Médica. Permite:

> Estimular el aprendizaje reflexivo, como condición para alcanzar el desarrollo de los estudiantes y estar en condiciones de actuar de manera integral en la solución de los problemas profesionales;

$>$ Hace posible que el contenido que se desarrolla, como objeto de aprendizaje, se estudie en sus contradicciones, las que al ser asumidas como problemas de la práctica médica;

$>$ Favorece la implicación productiva de los estudiantes en su aprendizaje;

> Facilita la búsqueda, el cuestionamiento, la puesta en práctica de procedimientos por parte de los estudiantes para encontrar la solución a los problemas profesionales;

$>$ Fomenta el vínculo teoría-práctica, al considerar el trabajo con problemas profesionales relacionados con la actividad laboral de los estudiantes, en una concepción de aprendizaje apoyada en la actividad reflexiva de los estudiantes, la problematización y la motivación;

> Concede una función protagónica a los estudiantes en su proceso de aprendizaje, en el que deben comprometerse no sólo con los resultados obtenidos sino, y de manera responsable, con las vías utilizadas para alcanzar las metas propuestas;

$>$ Convierte la clase (y otras formas organizativas) en escenario para la reflexión, el intercambio, la toma de decisiones y la generación de ideas nuevas, frutos del trabajo individual y colectivo que realizan los estudiantes, bajo la dirección del profesor;

> Exige que el control se efectúe sistemáticamente, en el que tengan primacía la autovaloración y la valoración colectiva, para comprobar los logros de los estudiantes en su aprendizaje;

> Permite que los estudiantes dominen la problemática que se les presentan desde la asignatura y aporten soluciones desde diferentes ópticas de análisis;

> Se apoya en el planteamiento y la solución de problemas, que deben partir de la realidad profesional en que se insertan los estudiantes en su actividad laboral, favoreciendo su preparación en y desde la práctica médica. 


\section{Descripción de las etapas:}

La estrategia fue estructurada en cuatro etapas interrelacionadas que, en su ejecución, expresan la dinámica del modelo.

Etapa 1: Diagnóstico de los estudiantes

Objetivo: Comprobar la situación del aprendizaje reflexivo en los estudiantes, jerarquizando el análisis del cumplimiento de las dimensiones y los indicadores determinados para este tipo de aprendizaje.

Acciones fundamentales:

- Elaboración de los instrumentos para la realización del diagnóstico.

- Realización del diagnóstico.

- Análisis de los resultados e identificación de las necesidades de los estudiantes.

Etapa 2: Planificación de la estrategia

Objetivo: Propiciar las condiciones necesarias para la puesta en práctica de la estrategia, a partir de los resultados del diagnóstico.

Acciones fundamentales:

- Preparación de los profesores en las características de la estrategia.

- Reflexión de los participantes sobre los requisitos determinados para diseñar las actividades.

- Selección de los procedimientos propuestos y su utilización, en correspondencia con el método seleccionado y las tareas docentes a ejecutar por los estudiantes.

Etapa 3: Implementación de la estrategia en el proceso de enseñanza-aprendizaje de la asignatura Genética Médica en los estudiantes de segundo año de la carrera de Medicina.

Objetivo: Ejecutar las diferentes acciones diseñadas en la planificación de la estrategia.

Esta etapa se ejecuta por los profesores y los estudiantes para lograr la participación cognoscitiva productiva de estos y garantizar que el aprendizaje sea reflexivo.

Acciones fundamentales:

- Conferencia introductoria.

- Actividades docentes problematizadoras.

- Valoración del trabajo que se realiza en la búsqueda de la solución de los problemas de la asignatura Genética Médica.

Etapa 4: Evaluación de la estrategia. (Autoperfeccionamiento de la estrategia)

Objetivo: Apreciar la marcha de la estrategia y realizar las correcciones que correspondan para su mejoramiento.

Acciones fundamentales:

- Análisis del desenvolvimiento de cada estudiante en los indicadores determinados para el aprendizaje reflexivo.

- Valoración de la manera en que los estudiantes incorporan lo aprendido a su actividad práctica.

- Reajuste de la estrategia, de acuerdo con los resultados obtenidos por los estudiantes.

La aplicación de la estrategia didáctica permitió alcanzar logros significativos en el desarrollo de un aprendizaje reflexivo de los estudiantes con los que se trabajó.

\section{Destacándose}

El contenido que se desarrolla, como objeto de aprendizaje, permitió analizar las contradicciones y presentar los problemas profesionales, que fueron resueltas en la práctica médica;

Participación activa en la solución de los problemas profesionales y mayor implicación productiva de los estudiantes en su aprendizaje; 
Enriquecimiento del vínculo teoría-práctica, basado en la actividad reflexiva de los estudiantes, la problematización y la motivación;

Mayor control sistemático de la valoración colectiva, de los logros de los estudiantes en su aprendizaje, el dominio de la problemática que se les presentan desde la asignatura y su aporte a las posibles soluciones con énfasis en la práctica laboral.

\section{CONCLUSIÓN}

El aprendizaje reflexivo en la formación de profesores ha sido considerado desde las primeras décadas del siglo pasado; no obstante, su puesta en práctica ha cobrado mayor auge en los últimos años, como respuesta a la necesidad de lograr un aprendizaje que conduzca al desarrollo y la transformación de los que aprenden en cualquier nivel de enseñanza, y con énfasis en la formación de profesionales para la salud, desde el vínculo indisoluble entre los componentes académico, laboral e investigativo, a partir de solucionar los problemas inherentes a esta profesión.

En la conducción del proceso de enseñanza aprendizaje de la Disciplina y asignatura Genética Médica, las dificultades en el aprendizaje reflexivo de los estudiantes no están dadas por las características propias del contenido, sino que son resultado de problemas metodológicos en su dirección.

Las actividades docentes que se desarrollan responden más al tradicionalismo que a las exigencias de este aprendizaje, en lo que influye, en gran medida, el poco conocimiento que sobre el aprendizaje reflexivo tienen los profesores y la manera de lograrlo en sus estudiantes.

El aprendizaje reflexivo requiere para su alcance que el profesor, en su condición de dirigente del proceso de enseñanza-aprendizaje, estructure su actividad de manera que los estudiantes solucionen problemas docentes surgidos a partir de las contradicciones inherentes a los componentes académico, laboral e investigativo, y que son reflejo de la vida cotidiana, para que asuman una posición de búsqueda, poniendo en función todos los recursos necesarios, tanto cognoscitivo-instrumentales como motivacional-afectivos, y en esta búsqueda de soluciones no solo encuentran nuevos contenidos, sino que se apropian de una metodología, que les permite llegar a plantearse nuevos problemas por sí mismos.

La estrategia didáctica, diseñada para el desarrollo del aprendizaje reflexivo, constituye una novedad en la enseñanza de la asignatura Genética Médica, cuya aplicación contribuye al desarrollo de este tipo de aprendizaje y a la formación de profesionales para la salud con una visión reflexiva de los problemas profesionales a atender durante su vida laboral.

\section{BIBLIOGRAFÍA}

1. Addine F, F (2006). Didáctica, Teoría y Práctica. La Habana: Editorial Pueblo y Educación; 2006

2. García B, G.(2010). Compendio de Pedagogía. La Habana: Editorial Pueblo y Educación.

3. Castellanos S (2003). D. Enseñar y Aprender en la escuela. La Habana: Editorial Pueblo y Educación.

4.

5. Diana Rosa Martín Sospedra \& José Ignacio Herrera Rodríguez. La formación de los docentes universitarios para potenciar el aprendizaje desarrollador. Gaceta Médica Espirituana 2014 [fecha de acceso 4 enero de 2015]; 16, (2): 1 a la 9. Recuperado de: http://revgmespirituana.sld.cu/index.php/gme/article/view/658/514

6. Martínez LI, M. (1998). Calidad educacional, actividad pedagógica y creatividad .La Habana: Editorial Academia.

7. Viera T, T. (2003). El aprendizaje verbal significativo de Ausubel. Algunas consideraciones desde el enfoque histórico. Revista Universidades.

8. Torrance, E.P., y Safter, H.T (1999). Making the creative leap beyond: Revision of the search for satori and creativity. Buffalo, NY: CEF Press 